\title{
SYMBOLIC DYNAMICS OF PIECEWISE CONTRACTIONS
}

\author{
Benito Pires \\ Departamento de Computação e Matemática, Faculdade de Filosofia, Ciências e Letras \\ Universidade de São Paulo, 14040-901, Ribeirão Preto - SP, Brazil \\ benito@usp.br
}

\begin{abstract}
A map $f:[0,1) \rightarrow[0,1)$ is a piecewise contraction of $n$ intervals $(n$-PC) if there exist $0<\lambda<1$ and a partition of $I=[0,1)$ into intervals $I_{1}, I_{2}, \ldots, I_{n}$ such that $|f(x)-f(y)| \leq \lambda|x-y|$ for every $x, y \in I_{i}(i=1,2, \ldots, n)$. An infinite word $\theta=\theta_{0} \theta_{1} \ldots$ over the alphabet $\mathcal{A}=\{1, \ldots, n\}$ is a natural coding of $f$ if there exists $x \in I$ such that $\theta_{k}=i$ whenever $f^{k}(x) \in I_{i}$. We prove that if $\theta$ is a natural coding of an injective $n$-PC, then some infinite subword of $\theta$ is either periodic or isomorphic to a natural coding of a topologically transitive $m$-interval exchange transformation ( $m$-IET), where $m \leq n$. Conversely, every natural coding of a topologically transitive $n$-IET is also a natural coding of some injective $n$-PC.
\end{abstract}

\section{INTRODUCTION}

Throughout this article, let $I=[0,1)$ denote the unit interval. A map $f: I \rightarrow I$ is a piecewise contraction of $n$ intervals $(n-\mathrm{PC})$ if there exist $0<\lambda<1$ and a partition of $I$ into non-degenerate intervals $I_{1}, \ldots, I_{n}$ such that $\left.f\right|_{I_{i}}$ is $\lambda$-Lipschitz for every $1 \leq i \leq n$. If, in particular, there exist $b_{1}, \ldots, b_{n} \in \mathbb{R}$ and $\sigma_{1}, \ldots, \sigma_{n} \in\{-1,1\}$ such that $f(x)=\sigma_{i} \lambda x+b_{i}$ for every $x \in I_{i}$, then we say that $f$ is a piecewise $\lambda$-affine contraction.

The natural $f$-coding of a point $x \in I$ is the infinite word $\theta_{f}(x)=\theta_{0} \theta_{1} \ldots$ defined by $\theta_{k}=i$ whenever $f^{k}(x) \in I_{i}$, where $f^{0}$ denotes the identity map. We say that an infinite word $\theta$ is a natural coding of $f$ if $\theta=\theta_{f}(x)$ for some $x \in I$. We say that $\theta$ is ultimately periodic (respectively, periodic) if there exist finite subwords $u, v$ of $\theta$ such that $\theta=u v v \ldots$ (respectively, $\theta=v v \ldots$ ). The language $\mathcal{L}(\theta)$ of a natural coding $\theta$ is the union of the sets $L_{k}(\theta)=\left\{\theta_{m} \theta_{m+1} \cdots \theta_{m+k-1}: m \geq 0\right\}$ of finite subwords of length $k$ occuring in $\theta$, where $L_{0}$ is the one-point-set formed by the empty word.

In this article, we give a complete and systematic description of the languages of injective $n$-PCs, $n \geq 2$, by providing a dictionary between these languages and the fairly well-understood languages of interval exchange transformations (IETs). We also provide converse results which enable us to construct $n$-PCS with any prescribed admissible coding.

2000 Mathematics Subject Classification. Primary 37E05, 37B10 Secondary 54H20.

Key words and phrases. Piecewise contraction, topological dynamics, symbolic dynamics. 
The first point addressed in this article consists in providing the list of all admissible natural codings of injective $n$-PCs. Natural codings of piecewise contractions defined on 2 intervals (or more generally, defined on 2 complete metric spaces) were provided by Gambaudo and Tresser [14] and are intrinsically related to natural codings of rotations of the circle. Concerning languages of injective $n$-PCs $f: I \rightarrow I$ for $n>2$, some progress was made recently by Catsigeras, Guiraud and Meyroneinc [7]. They proved that for each natural coding $\theta$ of $f$, the complexity function of the language $\mathcal{L}(\theta)$, defined by $p_{\theta}(k)=\# L_{k}(\theta)$, where \# denotes cardinality, is eventually affine.

The second point concerns the problem of how to construct $n$-PCs with any prescribed list of admissible natural codings. In this regard, it follows from the works [21, 22, 23] that a generic $n$-PC admits only ultimately periodic natural codings. Therefore, $n$-PCs with no ultimately periodic natural coding are exotic and their construction is a nontrivial issue. The existence of 2-PCs having no ultimately periodic natural coding is related to the existence of smooth flows on the 2-torus with pathological dynamics (see Cherry [8]). More generally, 2-PCs topologically semiconjugate to irrational rotations have being constructed and studied via a rotation number approach (see [4, [5, 6, 15, 16, 18]). Here we address the second point in full generality, by using another approach, based on the existence of an invariant measure (see [26]). In particular, we prove that every minimal $n$-IET, with $n \geq 2$, with or without flips, is a topological factor of an $n$-PC with no ultimately periodic natural coding. This combined with Keane's irrationality criteria [17, p. 27] provides a huge class of exotic $n$-PCs. Since every irrational rotation can be considered as a minimal 2-PC, the previous results fit into our framework.

As for the motivation to study $n$-PCs, it is worth remarking that they describe pretty well the dynamics of some Cherry flows on 2-manifolds, dissipative outer billiards, traffic systems, queueing systems and switched server systems.

Acknowledgments. The author is very grateful to Filipe Fernandes and Francisco Braun whose comments contributed to the improvement of the first version. The author was partially supported by grant \# 2018/06916-0, São Paulo Research Foundation (FAPESP) and by CNPq.

\section{Statement of the Results}

A bijective map $T: I \rightarrow I$ is an $n$-interval exchange transformation ( $n$-IET) if there exist a partition $I_{1}, \ldots, I_{n}$ of $I$ into non-degenerate intervals, $b_{1}, \ldots, b_{n} \in \mathbb{R}$ and $\sigma_{1}, \ldots, \sigma_{n} \in$ $\{-1,1\}$ such that $T(x)=\sigma_{i} x+b_{i}$ for every $x \in I_{i}$. an $n$-IET $T$ is standard if $I_{i}$ a leftclosed right-open interval and $\left.T\right|_{I_{i}}$ is the translation $x \mapsto x+b_{i}$ for every $1 \leq i \leq n$. Following [20], we say that a non-standard $n$-IET $T$ has flips if, for some $1 \leq i \leq n,\left.T\right|_{I_{i}}$ is the map $x \mapsto-x+b_{i}$. We say that an $n$-IET $T: I \rightarrow I$ is irreducible if there is no $0<\delta<1$ such that $T([0, \delta)) \subset[0, \delta)$ or, equivalently, if there is no $1 \leq j \leq n-1$ such that $T\left(\cup_{i=1}^{j} I_{i}\right) \subset \cup_{i=1}^{j} I_{i}$. 
An $n$-IET $T: I \rightarrow I$ is: topologically transitive if it has a dense $T$-orbit $\{x, T(x), \ldots\}$, minimal if every $T$-orbit is dense, and aperiodic if it has no periodic orbit. A periodic orbit $\gamma$ is attractive if there exists an open set $U_{\gamma}$ such that $\omega(x)=\gamma$ for every $x \in U_{\gamma}$. In a standard $n$-IET, every periodic orbit is attractive, thus in this case topological transitivity is equivalent to minimality. An $n$-IET $T$ satisfies the infinite distinct orbit condition (i.d.o.c.) if the orbits $\left\{x_{i}, T\left(x_{i}\right), \ldots\right\}$ of its discontinuities $x_{i}, 1 \leq i \leq n-1$, are infinite and pairwise disjoint. Keane [17] proved that every irreducible standard $n$-IET, $n \geq 2$, satisfying the i.d.o.c. is minimal. The natural T-coding of a point $x \in I$ is the infinite word $\theta_{T}(x)=\theta_{0} \theta_{1} \ldots$ defined by $\theta_{k}=i$ whenever $T^{k}(x) \in I_{i}$. If $T$ is an irreducible standard $n$-IET satisfying the i.d.o.c., then the language $\mathcal{L}(\theta)$ of a natural $T$-coding $\theta$ is the same for any $\theta$. In this case, we define the language of $T$, denoted by $\mathcal{L}$, to be the language $\mathcal{L}(\theta)$ of any of its natural $T$-codings.

The alphabet $\mathcal{A}(\theta)$ of an infinite word $\theta=\theta_{0} \theta_{1} \ldots$ is the set of letters that occur in $\theta$. We say that two infinite words $\theta=\theta_{0} \theta_{1} \ldots$ and $\omega=\omega_{0} \omega_{1} \ldots$ are isomorphic if their alphabets $\mathcal{A}(\theta)$ and $\mathcal{A}(\omega)$ have the same cardinality and there is a bijection $\pi: \mathcal{A}(\theta) \rightarrow \mathcal{A}(\omega)$ such that $\omega_{k}=\pi\left(\theta_{k}\right)$ for every $k \geq 0$. For example, the infinite words

$$
\theta=010010001 \ldots \text { and } \omega=121121112 \ldots
$$

are isomorphic because $\mathcal{A}(\theta)=\{0,1\}$ and $\mathcal{A}(\omega)=\{1,2\}$ have the same cardinality and $\omega_{k}=\pi\left(\theta_{k}\right)$ for every $k \geq 0$, where the bijection $\pi: \mathcal{A}(\theta) \rightarrow \mathcal{A}(\omega)$ is given by $\pi(0)=1$ and $\pi(1)=2$.

Our main results are the following.

Theorem 2.1. Let $f: I \rightarrow I$ be an injective $n-P C$, then there exist $2 \leq m \leq n$ and an $m$-IET T:I $\rightarrow I$ without attractive periodic orbits such that for each $x \in I$ there exists an integer $k \geq 0$ such that the natural $f$-coding of $f^{k}(x)$ is either periodic or isomorphic to a non ultimately periodic natural coding of $T$.

Theorem 2.2. Given any topologically transitive $n$-IET T: $I \rightarrow I$, there exist an injective piecewise $\frac{1}{2}$-affine contraction $f_{T}: I \rightarrow I$ of $n$ intervals and a continuous, surjective, nondecreasing map $h: I \rightarrow I$ such that $\theta_{f_{T}}(x)=\theta_{T}(h(x))$ for every $x \in I$. In particular, if $T$ is an irreducible standard n-IET satisfying the i.d.o.c., then the language of each natural coding of $f_{T}$ equals the language of $T$.

In Theorem 2.1, the term "without attractive periodic orbits" may be replaced by "aperiodic" in the case in which $I_{i}=\left[x_{i-1}, x_{i}\right)$ and $\left.f\right|_{I_{i}}, 1 \leq i \leq n$, is (strictly) increasing, where $I_{1}, \ldots, I_{n}$ is the partition associated to $f$.

Theorem 2.1 turns out to be a dictionary between languages of PCs and languages of IETs. Languages of minimal IETs were studied by Belov and Chernyat'ev [2], Ferenczi [11], Ferenczi and Zamboni [12, and Dolce and Perrin [10]. In particular, it is known that if $\theta$ is a natural coding of an irreducible standard minimal $n$-IET, then the language $\mathcal{L}(\theta)$ does not depend on $\theta$, is uniformly recurrent and has complexity function satisfying 
$p_{\theta}(k) \leq(n-1) k+1$, where the equality holds if the IET satisfies the i.d.o.c.. Languages generated by substitutions (e.g. languages of self-similar IETs) were studied by Lopez and Narbel [19]. Natural codings of aperiodic $n$-IETs are isomorphic to natural codings of topologically transitive $n$-IETs.

Theorem 2.2 provides examples of $n$-PCs without periodic or ghost orbits, or equivalently, without ultimately periodic natural codings. These examples are not easy to construct because generically $n$-PCs of the interval are asymptotically periodic (see [22, 23]). The following result is a corollary of Theorem 2.1.

Corollary 2.3. Let $\theta$ be a natural coding of an injective $n-P C$, then some infinite subword of $\theta$ is either periodic or isomorphic to a non ultimately periodic natural coding of a topologically transitive $m$-IET, where $2 \leq m \leq n$.

Theorems 2.1 and 2.2 imply, in particular, the result of Catsigeras, Guiraud and Meyroneinc [7] concerning the complexity function of languages of $n-\mathrm{PCs}$, which is stated below in a more complete way, with $f_{T}$ given by Theorem 2.2 .

Corollary 2.4. Let $\theta$ be a natural coding of an injective $n-P C f: I \rightarrow I$, then

(i) There exist $\alpha \in\{0,1, \ldots, n-1\}, \beta \geq 1$ and $k_{0} \geq 1$ such that the complexity function of $\theta$ satisfies $p_{\theta}(k)=\alpha k+\beta$ for every $k \geq k_{0}$ with $\beta=1$ if $\alpha=n-1$;

(ii) If $n \geq 2, T: I \rightarrow I$ is a standard $n$-IET satisfying the i.d.o.c. and $f=f_{T}$, then $p_{\theta}(k)=(n-1) k+1$ for every $k \geq 1$.

The particular family of 2-PCs $f: I \rightarrow I$ defined by $f(x)=\lambda x+\delta(\bmod 1)$ was considered by Bugeaud [4, 5, Bugeaud and Conze [6] and, more recently, by Janson and Öberg [16, and also by Laurent and Nogueira [18, by means of a rotation number approach. Concerning such family, we provide the following corollary, which turns out to be a special case of [18, Corollary 7]. We recall that an $n$-PC $f: I \rightarrow I$ is topologically semiconjugate to an $n$-IET $T: I \rightarrow I$ if there exists a continuous, nondecreasing and surjective map $h: I \rightarrow I$ such that $h \circ f=T \circ h$.

Corollary 2.5. For each irrational $0<\alpha<1$, there exists a transcendent $\delta \in \mathbb{R}$ such that the 2-PC $f_{T}: I \rightarrow I$ and the minimal 2-IET T:I $\rightarrow I$ defined by

$$
f_{T}(x)=\frac{1}{2} x+\delta(\bmod 1) \quad \text { and } \quad T(x)=x+\alpha(\bmod 1)
$$

are topologically semiconjugate and every natural coding of $f_{T}$ is a Sturmian sequence. In particular, if $\alpha=2-\varphi$, where $\varphi=(1+\sqrt{5}) / 2$ is the golden ratio, then $\delta=1-\frac{R}{2}$, where $R$ is the rabbit constant.

In Corollary 2.5, we have that $\delta=\frac{1}{4} \sum_{k \geq 0} \theta_{k} 2^{-k}$, where $\theta=\theta_{0} \theta_{1} \ldots$ is the natural coding of $\alpha$ under the action of the irrational rotation $x \mapsto x+\alpha(\bmod 1)$. 


\section{Preparatory Lemmas}

In this section, we present some results that will be used to prove Theorem 2.1, as the next table clarifies.

\begin{tabular}{l|l}
\hline result & needs \\
\hline Lemma 3.3 & Lemma 3.2 \\
Corollary 3.4 & Lemma 3.3 \\
Theorem 3.5 & Lemma 3.6 and [26, Theorem 2.1] \\
Theorem 2.1] & Lemma 3.1, Corollary 3.4 and Theorem 3.5 \\
\hline
\end{tabular}

Throughout this section, let $I=[0,1)$ and $f: I \rightarrow I$ be an injective $n$-PC with associated partition $I_{1}, \ldots, I_{n}$ whose endpoints are $0=x_{0}<x_{1}<\cdots<x_{n}=1$.

The $\omega$-limit set of $x \in I$ is defined by

$$
\omega(x)=\bigcap_{\ell \geq 1} \overline{\bigcup_{k \geq \ell}\left\{f^{k}(x)\right\}},
$$

where $\bar{S}$ denotes the topological closure in $\mathbb{R}$ of any set $S \subset I$.

Lemma 3.1. Let $x \in I$ be such that $\omega(x)$ is finite, then there exists an integer $k \geq 0$ such that the natural $f$-coding of $f^{k}(x)$ is periodic.

Proof. We may assume that $\left\{x, f(x), f^{2}(x), \ldots\right\}$ is an infinite set, otherwise $x$ would be a periodic point, then we could take $k=0$. Since $\omega(x)$ is a finite set, we may write $\omega(x)=\left\{p_{1}, \ldots, p_{r}\right\}$. Without loss of generality, we suppose that $\omega(x) \subset(0,1)$, thus there exists $\epsilon>0$ so small that

$$
\epsilon<\frac{1}{4} \min _{1 \leq i<j \leq r}\left|p_{i}-p_{j}\right| \text { and } \bigcup_{j=1}^{r}\left(p_{j}-\epsilon, p_{j}\right) \cup\left(p_{j}, p_{j}+\epsilon\right) \subset I \backslash\left\{x_{0}, x_{1}, \ldots, x_{n-1}\right\} .
$$

In particular, if

$$
\mathscr{I}=\left\{\left(p_{1}-\epsilon, p_{1}\right),\left(p_{1}, p_{1}+\epsilon\right), \ldots,\left(p_{r}-\epsilon, p_{r}\right),\left(p_{r}, p_{r}+\epsilon\right)\right\},
$$

then $f(J)$ is an open interval for every $J \in \mathscr{I}$.

Let $\mathscr{I}^{\prime} \subset \mathscr{I}$ denote the subcollection formed by the intervals that are visited infinitely many times by the $f$-orbit of $x$, that is,

$$
\mathscr{I}^{\prime}=\left\{J \in \mathscr{I}:\left\{x, f(x), f^{2}(x), \ldots\right\} \cap J \text { is an infinite set }\right\} .
$$

We claim that for each $J_{1} \in \mathscr{I}^{\prime}$, there exists $J_{2} \in \mathscr{I}^{\prime}$ such that $f\left(J_{1}\right) \subset J_{2}$. Without loss of generality, suppose that $J_{1}=(p-\epsilon, p)$, where $p \in \omega(x)$. As $J_{1} \subset I \backslash\left\{x_{0}, x_{1}, \ldots, x_{n-1}\right\}$, we have that $\left.f\right|_{J_{1}}$ is a contraction, thus $f\left(J_{1}\right)$ is an open interval of length smaller than $\epsilon$. On the other hand, since $J_{1} \in \mathscr{I}^{\prime}$, there exists an increasing sequence of integers $0 \leq$ $k_{1}<k_{2}<\cdots$ such that $\left\{f^{k_{j}}(x)\right\}_{j \geq 1} \subset J$. Notice that $\lim _{j \rightarrow \infty} f^{k_{j}}(x)=p$, otherwise there would exist a point of $\omega(x)$ in $J_{1}$ different from $p$, which contradicts the first inequality in the definition of $\epsilon$. Because $\left.f\right|_{J_{1}}$ is injective and continuous, we have that $\left\{f^{k_{j}+1}(x)\right\}_{j \geq 1} \subset$ 
$f\left(J_{1}\right)$ converges to some point $q \in \omega(x) \cap \partial f\left(J_{1}\right)$, where $\partial f\left(J_{1}\right)$ denotes the endpoints of the open interval $f\left(J_{1}\right)$. Putting it all together, we conclude that $f\left(J_{1}\right)$ is an open interval that contains infinitely many points of the $f$-orbit of $x$, has length smaller than $\epsilon$, and has an endpoint in $\omega(x)$. Therefore, there exists $J_{2} \in \mathscr{I}^{\prime}$ such that $f\left(J_{1}\right) \subset J_{2}$.

To finish the proof, let $J \in \mathscr{I}^{\prime}$, then there exists $k^{\prime} \geq 0$ such that $f^{k^{\prime}}(x) \in J$. By the claim, there exist $1 \leq i_{1}<i_{2}$ and intervals $J_{1}, \ldots, J_{i_{1}}, J_{i_{1}+1}, \ldots, J_{i_{2}} \in \mathscr{I}^{\prime}$ such that $J_{1}=\mathrm{J}$, $J_{i_{1}}=J_{i_{2}}$ and $f\left(J_{i}\right) \subset J_{i+1}$ for all $1 \leq i \leq i_{2}-1$, proving that $f^{k}(x)$ has a periodic natural $f$-coding for some $k \geq k^{\prime}$.

Lemma 3.2. Let $J \subset I$ be an open interval, then there exists a finite set $B \subset I$ such that if $J_{0} \subset J \backslash B$ is an open interval, then one of the following happens:

(i) $f\left(J_{0}\right), f^{2}\left(J_{0}\right), \ldots$ are pairwise disjoint open intervals contained in $I \backslash J$;

(ii) $\exists m \geq 0$ such that $f^{m+1}\left(J_{0}\right)$ is open subinterval of $J$. Moreover, if $m \geq 1$, then $f\left(J_{0}\right), \ldots, f^{m}\left(J_{0}\right)$ are open subintervals of $I \backslash\left(J \cup\left\{x_{0}, x_{1}, \ldots, x_{n-1}\right\}\right)$.

Proof. Let $J \subset I$ be an open interval. Given $x \in I$, set

$$
\tau_{x}=\min \left\{k \geq 0: f^{-k}(\{x\}) \subset J\right\},
$$

where by convention $\inf \emptyset=\infty$. Let

$$
B=\bigcup\left\{f^{-\tau_{x}}(\{x\}): x \in\left\{x_{0}, x_{1}, \ldots, x_{n-1}\right\} \cup \partial J \text { and } \tau_{x}<\infty\right\} .
$$

Let $J_{0} \subset J \backslash B$ be an open interval, then one of the following alternatives happens: $\left\{f^{k}\left(J_{0}\right)\right\}_{k \geq 1} \subset I \backslash J$ or there exists an integer $\ell \geq 1$ such that $f^{\ell}\left(J_{0}\right) \cap J \neq \emptyset$. In the first case, by the injectivity of $f$ and also because $J_{0} \subset J \backslash B$, we have that $J_{0}, f\left(J_{0}\right)$, $f^{2}\left(J_{0}\right), \ldots$ are pairwise disjoint sets contained in $I \backslash\left\{x_{0}, x_{1}, \ldots, x_{n-1}\right\}$. Since each $\left.f\right|_{I_{i}}$ is Lipschitz continuous, we conclude that $f^{k}\left(J_{0}\right)$ is an open interval for every $k \geq 0$, which proves $(i)$. As for the second alternative, let $m=\min \left\{\ell \geq 1: f^{\ell}\left(J_{0}\right) \cap J \neq \emptyset\right\}-1$. If $m=0$, then $f\left(J_{0}\right) \cap J \neq \emptyset$, which together with the fact that $J_{0} \subset J \backslash B$ implies that $f\left(J_{0}\right)$ is an open subset of $J$. Otherwise, if $m \geq 1$, then proceeding as in the first case yields that the sets $f\left(J_{0}\right), \ldots, f^{m}\left(J_{0}\right)$ are pairwise disjoint open subintervals of $I \backslash\left(J \cup\left\{x_{0}, x_{1}, \ldots, x_{n-1}\right\}\right)$. Moreover, because $J_{0} \subset J \backslash B$, we have that $f^{m+1}\left(J_{0}\right) \cap J \neq \emptyset$ implies that $f^{m+1}\left(J_{0}\right)$ is an open subinterval of $J$.

Remark. The item (ii) of Lemma 3.2 implies that $\left.f^{m+1}\right|_{J_{0}}: J_{0} \mapsto f^{m+1}\left(J_{0}\right)$ is a bijective contraction.

Lemma 3.3. If for some $x \in I$ and $1 \leq i \leq n$, the set $\left\{x, f(x), f^{2}(x), \ldots\right\} \cap\left(x_{i-1}, x_{i}\right)$ is infinite and $\omega(x) \cap\left(x_{i-1}, x_{i}\right)=\emptyset$, then $\omega(x)$ is finite.

Proof. By hypothesis, we have that

(H1) $\left\{x, f(x), f^{2}(x), \ldots\right\} \cap\left(x_{i-1}, x_{i}\right)$ is infinite;

(H2) $\left\{x, f(x), f^{2}(x), \ldots\right\} \cap K$ is finite for all compact set $K \subset\left(x_{i-1}, x_{i}\right)$. 
By (H1), the orbit of $x$ returns to $J=\left(x_{i-1}, x_{i}\right)$ infinitely many times. Let $1 \leq k_{1}<k_{2}<$ ‥ denote the return times of $x$ to $J$ under the action of $f$. Because of (H2), we have only three cases to consider.

Case (a). $\lim _{j \rightarrow \infty} f^{k_{j}}(x)=x_{i-1}$.

Let $B$ the finite set given by Lemma 3.2 considering $J=\left(x_{i-1}, x_{i}\right)$. Let $\epsilon>0$ be so small that $J_{0}=\left(x_{i-1}, x_{i-1}+\epsilon\right)$ is a subset of $J \backslash B$. Notice that the alternative $(i)$ of Lemma 3.2 cannot occur. In fact, since $f^{k_{j}}(x) \downarrow x_{i-1}$, we have that $f^{k}\left(J_{0}\right) \cap J \neq \emptyset$ for many positive values of $k$. By exclusion, the item (ii) of Lemma 3.2 is true, then there exists $m \geq 0$ such that $f^{m+1}\left(J_{0}\right)$ is an open subinterval of $J$ and, if $m \geq 1$, then $f\left(J_{0}\right), \ldots, f^{m}\left(J_{0}\right)$ are open subintervals of $I \backslash\left(J \cup\left\{x_{0}, x_{1}, \ldots, x_{n-1}\right\}\right)$. In particular, if $y \in J_{0}$, then $m+1$ is the first return time of $y$ to $J$. This means that if $j_{0} \geq 1$ is such that $\left\{f^{k_{j}}(x)\right\}_{j \geq j_{0}} \subset J_{0}$, then $\left\{f^{k_{j}}(x)\right\}_{j>j_{0}} \subset f^{m+1}\left(J_{0}\right)$, implying that $x_{i-1}$ belongs to the boundary of the open interval $f^{m+1}\left(J_{0}\right)$. Moreover, since $\left.f^{m+1}\right|_{J_{0}}: J_{0} \rightarrow f^{m+1}\left(J_{0}\right)$ is a bijective contraction (see the Remark after Lemma 3.2 , we have that $f^{m+1}\left(J_{0}\right) \subset J$ is an open interval with length smaller than $\epsilon$ and with an endpoint in $x_{i-1}$, thus $f^{m+1}\left(J_{0}\right) \subset\left(x_{i-1}, x_{i-1}+\epsilon\right)=J_{0}$. This implies that $\omega(x)$ is finite.

Case (b). $\lim _{j \rightarrow \infty} f^{k_{j}}(x)=x_{i}$.

Just proceed as in Case (a) considering now $J_{0}=\left(x_{i}-\epsilon, x_{i}\right)$.

Case (c). $\bigcap_{\ell \geq 1} \overline{\bigcup_{j \geq \ell}\left\{f^{k_{j}}(x)\right\}}=\left\{x_{i-1}, x_{i}\right\}$.

The proof presented here is a variation of that used in Case (a). Let $\epsilon>0$ be so small that $J_{0}^{\prime}=\left(x_{i-1}, x_{i-1}+\epsilon\right)$ and $J_{0}^{\prime \prime}=\left(x_{i}-\epsilon, x_{i}\right)$ are contained in $J \backslash B$. Then by the same arguments used in Case (a), there exist $m^{\prime}, m^{\prime \prime} \geq 0$ such that $f^{m^{\prime}+1}\left(J_{0}^{\prime}\right)$ and $f^{m^{\prime \prime}+1}\left(J_{0}^{\prime \prime}\right)$ are disjoint open subintervals of $J$ and, if $m^{\prime} \geq 1$ (respectively, $m^{\prime \prime} \geq$ 1 ), then $f\left(J_{0}^{\prime}\right), \ldots, f^{m^{\prime}}\left(J_{0}^{\prime}\right)$ (respectively, $f\left(J_{0}^{\prime \prime}\right), \ldots, f^{m^{\prime \prime}}\left(J_{0}^{\prime \prime}\right)$ ) are open subintervals of $I \backslash\left(J \cup\left\{x_{0}, x_{1}, \ldots, x_{n-1}\right\}\right)$. In particular, if $y \in J_{0}^{\prime}$ (respectively, if $y \in J_{0}^{\prime \prime}$ ), then $m^{\prime}+1$ (respectively, $m^{\prime \prime}+1$ ) is the first return time of $y$ to $J$. This means that if $j_{0} \geq 1$ is such that $\left\{f^{k_{j}}(x)\right\}_{j \geq j_{0}} \subset J_{0}^{\prime} \cup J_{0}^{\prime \prime}$, then $\left\{f^{k_{j}}(x)\right\}_{j>j_{0}} \subset f^{m^{\prime}+1}\left(J_{0}^{\prime}\right) \cup f^{m^{\prime \prime}+1}\left(J_{0}^{\prime \prime}\right)$, implying that $x_{i-1} \in \partial f^{m^{\prime \prime}+1}\left(J_{0}^{\prime \prime}\right)$ and $x_{i} \in \partial f^{m^{\prime}+1}\left(J_{0}^{\prime}\right)$. Moreover, since $\left.f^{m^{\prime}+1}\right|_{J_{0}^{\prime}}: J_{0}^{\prime} \rightarrow f^{m^{\prime}+1}\left(J_{0}^{\prime}\right)$ and $\left.f^{m^{\prime \prime}+1}\right|_{J_{0}^{\prime \prime}}: J_{0}^{\prime \prime} \rightarrow f^{m^{\prime \prime}+1}\left(J_{0}^{\prime \prime}\right)$ are bijective contractions, we can argue in the same way as in Case (a) to conclude that $f^{m^{\prime}+1}\left(J_{0}^{\prime}\right) \subset J_{0}^{\prime \prime}$ and $f^{m^{\prime \prime}+1}\left(J_{0}^{\prime \prime}\right) \subset J_{0}^{\prime}$, proving that $\omega(x)$ is finite.

Lemma 3.3 leads to the following result. 
Corollary 3.4. Let $x \in I$ be such that $\omega(x)$ is infinite. If for some $1 \leq i \leq n$, the set $\left\{x, f(x), f^{2}(x), \ldots\right\} \cap\left(x_{i-1}, x_{i}\right)$ is infinite, then $\omega(x) \cap\left(x_{i-1}, x_{i}\right) \neq \emptyset$.

We will also need the following result, which is a variation of [26, Theorem 2.1].

Theorem 3.5. Let $x \in I$ be such that $\Lambda=\omega(x)$ is infinite, then there exists a non-atomic $f$-invariant Borel probability measure whose support is $\Lambda$.

The proof of Theorem 3.5 depends on Lemma 3.6 stated below. In what follows, let $x \in I$ be such that $\Lambda=\omega(x)$ is infinite. As $x$ is not periodic, there exists $\ell \geq 0$ such that $\left\{f^{k}(x): k \geq \ell\right\} \cap\left\{x_{0}, x_{1}, \ldots, x_{n-1}\right\}=\emptyset$. Hence, by replacing $x$ by $f^{\ell}(x)$ if necessary, we assume that

$$
\left\{x, f(x), f^{2}(x), \ldots\right\} \cap\left\{x_{0}, x_{1}, \ldots, x_{n-1}\right\}=\emptyset .
$$

Denote by $\left\{\nu_{m}\right\}_{m \geq 1}$ the sequence of Borel probability measures on $I$ defined by

$$
\nu_{m}=\frac{1}{m} \sum_{k=0}^{m-1} \delta_{f^{k}(x)},
$$

where $\delta_{f^{k}(x)}$ is the Dirac probability measure on $I$ concentrated at $f^{k}(x)$. By the BanachAlaoglu Theorem, there exist a Borel probability measure on $I$, denoted henceforth by $\nu$, and a subsequence of $\left\{\nu_{m}\right\}_{m \geq 1}$, denoted henceforth by $\left\{\nu_{m_{j}}\right\}_{j \geq 1}$, that converges to $\nu$ in the weak ${ }^{\star}$ - topology. We will keep these notations until the end of this section.

Lemma 3.6. Let $y \in I$, then there exist an open subinterval $J_{y}$ of I containing $y$ and an integer $j_{0} \geq 1$ such that $\nu_{m_{j}}\left(J_{y}\right)<\epsilon$ for every $j \geq j_{0}$. Moreover, the support of $\nu$ is $\Lambda=\omega(x)$.

Proof. Let $y \in I$ and $\epsilon>0$. We will prove that there exist $\delta>0$ and $j_{0} \geq 1$ such that the interval

$$
J_{y}= \begin{cases}{[0, \delta)} & \text { if } y=0 \\ (y-\delta, y+\delta) & \text { if } y>0\end{cases}
$$

satisfies $J_{y} \subset I$ and $\nu_{m_{j}}\left(J_{y}\right)<\epsilon$ for all $j \geq j_{0}$. Without loss of generality, we may assume that $y>0$ and $J_{y}=(y-\delta, y+\delta)$. Since $\nu$ is a probability measure, $\nu$ has at most countably many atoms, which means that the set

$$
\Delta=\{0<\delta<\min \{y, 1-y\}: \nu(\{y-\delta, y+\delta\})=0\}
$$

contains arbitrarily small values of $\delta$. It follows from [25, Theorem 6.1, p. 40] that if $\delta \in \Delta$, then

$$
J_{y} \subset I \quad \text { and } \quad \nu\left(J_{y}\right)=\lim _{j \rightarrow \infty} \nu_{m_{j}}\left(J_{y}\right) .
$$

Now have two cases to consider.

Case I : $y \notin \Lambda$, that is, $y \notin \omega(x)$. 
In this case, there exist $\delta \in \Delta$ and $j_{0} \geq 1$ such that $f^{k}(x) \notin J_{y}$ for every $k \geq m_{j_{0}}$. Let $j_{1} \geq j_{0}$ be such that $m_{j}>m_{j_{0}} / \epsilon$ for every $j \geq j_{1}$, then

$$
\nu_{m_{j}}\left(J_{y}\right)=\frac{1}{m_{j}} \#\left\{0 \leq k \leq m_{j_{0}}-1: f^{k}(x) \in J_{y}\right\} \leq \frac{m_{j_{0}}}{m_{j}}<\epsilon, \quad \forall j \geq j_{1} .
$$

Moreover, making $j \rightarrow \infty$ and using (3) yield $\nu\left(J_{y}\right)=0$, implying that $y$ does not belong to the support of $\nu$.

Case II $: y \in \Lambda$.

First assume that there exists an increasing sequence of integers $1 \leq k_{1}<k_{2}<\cdots$ such that $f^{k_{j}}(x) \uparrow y$. Since $f$ is an injective piecewise contraction, the following limits are well-defined:

$$
y_{0}=y, \quad y_{1}=\lim _{j \rightarrow \infty} f\left(f^{k_{j}}(x)\right), \quad y_{2}=\lim _{j \rightarrow \infty} f^{2}\left(f^{k_{j}}(x)\right), \quad \ldots
$$

We claim that $\#\left\{k \geq 1: y_{k}=y\right\} \leq 1$. By way of contradiction, suppose that there exist $1 \leq p<q$ such that $y_{q}=y_{p}=y$. It is elementary to see that for every $\delta>0$ small enough and $A_{0}=(y-\delta, y)$, the sets $A_{1}=f\left(A_{0}\right), A_{2}=f^{2}\left(A_{0}\right), \ldots, A_{q}=f^{q}\left(A_{0}\right)$ are open intervals of length less than $\delta$. Yet, $y_{k} \in \partial A_{k}$ for every $0 \leq k \leq q$. Hence, either $A_{p} \subset A_{0}$ or $A_{q} \subset A_{0}$, which contradicts the fact that $\omega(x)$ is infinite. In this way, the claim is true. Then, there exists $r_{0} \geq 1$ such that $y_{k} \neq y$ for all $k \geq r_{0}$. In particular, given $r \geq 1$, there exists $\delta_{1}=\delta_{1}(r)$ such that for every $0<\delta<\delta_{1}$,

$$
\#\left\{0 \leq k \leq r-1: f^{k}((y-\delta, y)) \cap J_{y} \neq \emptyset\right\} \leq 2 .
$$

Let $r>0$ be such that $\frac{3}{r}<\frac{\epsilon}{3}$. Set $\delta_{1}=\delta_{1}(r)$. Then, for all $0<\delta<\delta_{1}$ with $\delta \in \Delta$ and for any $j$ large enough,

$$
\nu_{m_{j}}((y-\delta, y))=\frac{1}{m_{j}} \#\left\{0 \leq k \leq m_{j}-1: f^{k}(x) \in J_{y}\right\} \leq \frac{3}{r}<\frac{\epsilon}{3} .
$$

Now assume that the sequence $1 \leq k_{1}<k_{2}<\ldots$ does not exist, then for every $\delta$ small enough,

$$
\nu_{m_{j}}((y-\delta, y))=0<\frac{\epsilon}{3}
$$

Likewise, there exists $\delta_{2}>0$ such that for all $0<\delta<\delta_{2}$ with $\delta \in \Delta$, we have that $\nu_{m_{j}}((y, y+\delta))<\frac{\epsilon}{3}$ for any $j$ large enough. Moreover, $\nu_{m_{j}}(\{y\})<\frac{\epsilon}{3}$ for any $j$ large enough. Putting all together, there exist $\delta>0$ with $\delta \in \Delta$ and $j_{0} \geq 1$ such that $\nu_{m_{j}}\left(J_{y}\right)<\epsilon$ for all $j \geq j_{0}$.

It remains to prove that in this case $y$ belongs to the support of $\nu$. By the above, we know that the orbit of $x$ enters in $J_{y}$ infinitely many times. If we prove that the return times of $x$ to $J_{y}$ are bounded, then we will conclude that $\inf _{j \geq j_{0}} \nu_{m_{j}}\left(J_{y}\right)>0$, which together with (3) will imply that $\nu\left(J_{y}\right)>0$. Let $S=\left\{x_{0}, x_{1}, \ldots, x_{n-1}\right\} \cup \partial J_{y}$ and $S^{\prime}=\left\{z \in S: \cup_{k \geq 0} f^{-k}(\{z\}) \cap J_{y} \neq \emptyset\right\}$. Given $z \in S^{\prime}$, let

$$
\tau_{z}=\min \left\{k \geq 0: f^{-k}(\{z\}) \subset J_{y}\right\}
$$


and $B=\left\{f^{-\tau_{z}}(z): z \in S^{\prime}\right\}$. If $U$ is a connected component of $J_{y} \backslash B$, then all points of $U$ either never return to $J_{y}$ or return to $J_{y}$ at the same time. The second case always happens when $U \subset J_{y}$ is a small interval with an endpoint at $y$. In particular, the return times of the points of the orbit of $x$ to $J_{y}$ are bounded.

Proof of Theorem 3.5. Theorem 3.5] is a variation of [26, Theorem 2.1] where the hypotheses of no connection and no periodic orbit were weakened. Here we just point out which change is necessary in the proof of [26, Theorem 2.1]. In this regard, [26, Lemma 3.2] ought to be replaced by Lemma 3.6. The hypothesis that $f$ has no periodic orbit in the statement of [26, Theorem 2.1] is not necessary: all we need is that $\omega(x)$ is infinite. In this way, the claims of [26, Theorem 2.1] hold in our context, which proves Theorem 3.5 .

\section{Proof of Theorem 2.1}

Throughout this section, let $f: I \rightarrow I$ be an $n$-PC with associated partition $I_{1}, \ldots, I_{n}$ having endpoints $0=x_{0}<x_{1}<\cdots<x_{n}=1$. We will need the following elementary result.

Lemma 4.1 ([21, Lemma 3.6]). There exist $r \leq 2 n$ pairwise disjoint open intervals $F_{1}, \ldots, F_{r}$ such that $f^{k}\left(F_{j}\right), 1 \leq j \leq r, k<0$, are empty sets, and $f^{k}\left(F_{j}\right), 1 \leq j \leq r$, $k \geq 0$, are pairwise disjoint open intervals and $\Omega=\cup_{j=1}^{r} \cup_{k \geq 0} f^{k}\left(F_{j}\right)$ is a dense subset of $I \backslash\left\{x_{0}, x_{1}, \ldots, x_{n-1}\right\}$ having Lebesgue measure 1 .

A non-empty compact subset $\Lambda \subset[0,1]$ is an attractor of $f$ if there exists $p \in I$ such that $\Lambda=\omega(p)$. Let $F_{1}, \ldots, F_{r}$ be as in the statement of Lemma 4.1, then for each $1 \leq j \leq r, \cup_{k \geq 0} f^{k}\left(F_{j}\right) \cap\left\{x_{0}, x_{1}, \ldots, x_{n-1}\right\}=\emptyset$, implying that $\omega\left(p_{j}\right)$ is the same for any $p_{j} \in \cup_{k \geq 0} f^{k}\left(F_{j}\right)$. In this way, the attractors

$$
\Lambda_{1}=\omega\left(p_{1}\right), \ldots, \Lambda_{r}=\omega\left(p_{r}\right)
$$

do not depend on the choice of $\left(p_{1}, \ldots, p_{r}\right) \in F_{1} \times \cdots \times F_{r}$.

Lemma 4.2. Let $p \in I$. If $\omega(p)$ is infinite, then $\omega(p) \in \Lambda_{1} \cup \cdots \cup \Lambda_{r}$.

Proof. Since $\omega(p)$ is infinite, the $f$-orbit of $p$ is not periodic. In particular, there exists $k_{0} \geq 0$ such that the $f$-orbit of $f^{k_{0}}(p)$ does not pass through discontinuities. By the density of $\Omega$, there exists $1 \leq j \leq r$ such that $f^{k_{0}}(p) \in \omega\left(p_{j}\right)$. Then, $\omega(p)=\omega\left(f^{k_{0}}(p)\right) \subset$ $\omega\left(p_{j}\right)=\Lambda_{j}$.

Without loss of generality, by replacing $r$ by a smaller number, we may assume that the sets $\Lambda_{1}, \ldots, \Lambda_{r}$ are pairwise distinct. It follows from Lemma 4.1 that $S=I \backslash \Omega$ is a Lebesgue null set. Let $1 \leq j \leq r$. As $\bar{S}=S \cup\{1\}$ and $\Lambda_{j} \subset \bar{S}$, we have that $\bar{S}$ has empty interior, hence $\Lambda_{j}$ is totally disconnected. By the Cantor-Bendixson Theorem, we conclude that $\Lambda_{j}$ is either a finite set or the union of a Cantor set with a discrete set. If all the attractors $\Lambda_{1}, \ldots, \Lambda_{r}$ are finite, then, by Lemmas 3.1 and 4.2 , all natural codings of $f$ are ultimately periodic and we are done. Otherwise, there are $1 \leq s \leq r$ infinite 
attractors. Without loss of generality, assume that $\Lambda_{1}, \ldots, \Lambda_{s}$ are the infinite attractors. It follows from Theorem 3.5 that for each $1 \leq j \leq s$, there exists a non-atomic $f$-invariant Borel probability measure $\mu_{j}$ whose support is $\Lambda_{j}$. Hence, if

$$
\mu=\frac{1}{s} \mu_{1}+\cdots+\frac{1}{s} \mu_{s}, \quad A=\Lambda_{1} \cup \cdots \cup \Lambda_{s},
$$

then $\mu$ is a non-atomic $f$-invariant Borel probability measure with support equal to $A$.

Let $h:[0,1] \rightarrow[0,1]$ be the nondecreasing, continuous, surjective map defined by $h(t)=$ $\mu([0, t]), t \in I$. Notice that $h$ is strictly increasing on $A$ and constant on each connected component of $I \backslash A$. Given $x, x^{\prime} \in I_{i}$ with $h(x)=h\left(x^{\prime}\right)$, we claim that $h(f(x))=h\left(f\left(x^{\prime}\right)\right)$. Since $f$ is injective, $\left.f\right|_{I_{i}}$ is either increasing or decreasing. Without loss of generality, in what follows, assume that $\left.f\right|_{I_{i}}$ is increasing (and continuous) for every $1 \leq i \leq n$. Assume $x \leq x^{\prime}$, then $f(x) \leq f\left(x^{\prime}\right)$. Moreover, since $\left.f\right|_{I_{i}}$ is increasing and continuous,

$$
\left[x, x^{\prime}\right)=f^{-1}\left(\left[f(x), f\left(x^{\prime}\right)\right)\right) .
$$

Hence, since $\mu$ is non-atomic and $f$-invariant, we have that for any $x \leq x^{\prime}$ in $I_{i}$,

$$
h\left(f\left(x^{\prime}\right)\right)-h(f(x))=\mu\left(\left[f(x), f\left(x^{\prime}\right)\right)\right)=\mu\left(f^{-1}\left(\left[f(x), f\left(x^{\prime}\right)\right)\right)\right)=h\left(x^{\prime}\right)-h(x),
$$

which proves the claim.

We will use (5) to define an IET $T: I \rightarrow I$. Let

$$
\mathcal{I}=\left\{1 \leq i \leq n:\left(x_{i-1}, x_{i}\right) \cap A \neq \emptyset\right\}
$$

where $x_{i-1}$ and $x_{i}$ are the endpoints of $I_{i}$. Let $m \leq n$ be the cardinality of $\mathcal{I}$, then we may write $\mathcal{I}=\left\{i_{1}, \ldots, i_{m}\right\}$. Let $0=y_{0}<y_{1}<\cdots<y_{m}=1$ be the points defined by $y_{\ell}=h\left(x_{i_{\ell}}\right), 1 \leq \ell \leq m$. Let $T: I \rightarrow I$ be the map that at $h(x) \in I \backslash\left\{y_{0}, y_{1}, \ldots, y_{m-1}\right\}$ takes the value

$$
T(h(x))=h(f(x)) .
$$

The map $T$ is well-defined on $I \backslash\left\{y_{0}, y_{1}, \ldots, y_{m-1}\right\}$. To see that, let $x, x^{\prime} \in I, x<x^{\prime}$, be such that $h(x)=h\left(x^{\prime}\right)$ is a point in $I \backslash\left\{y_{0}, y_{1}, \ldots, y_{m-1}\right\}$. Then, $\left\{x, x^{\prime}\right\} \subset \bigcup_{i \in \mathcal{I}}\left(x_{i-1}, x_{i}\right)$, otherwise $x$ or $x^{\prime}$ would belong to $\left\{y_{0}, y_{1}, \ldots, y_{m-1}\right\}$. In this way, there exist $i, j \in \mathcal{I}$ such that $x \in\left(x_{i-1}, x_{i}\right)$ and $x^{\prime} \in\left(x_{j-1}, x_{j}\right)$. If $i \neq j$, then the hypothesis $h(x)=h\left(x^{\prime}\right)$ yields $h(x)=h\left(x_{i}\right)=h\left(x_{j-1}\right)=h\left(x^{\prime}\right)$, showing that $h(x) \in\left\{y_{0}, y_{1}, \ldots, y_{m-1}\right\}$, which is a contradiction. Hence, the only alternative left is $i=j$ and $x, x^{\prime} \in\left(x_{i-1}, x_{i}\right)$. In this way, $x, x^{\prime}$ belong to the same interval $I_{i}$ and (5) implies that $T(h(x))=T\left(h\left(x^{\prime}\right)\right)$, thus $T$ is well-defined on $I \backslash\left\{y_{0}, y_{1}, \ldots, y_{m-1}\right\}$.

Let us prove that $\left.T\right|_{\left(y_{\ell-1}, y_{\ell}\right)}, 1 \leq \ell \leq m$, is a translation. If $y, y^{\prime}$ are two points in $\left(y_{\ell-1}, y_{\ell}\right)$, there exist $x, x^{\prime} \in\left(x_{i_{\ell}-1}, x_{i_{\ell}}\right)$ such that $y=h(x)$ and $y^{\prime}=h\left(x^{\prime}\right)$, then (5) and (6) yield

$$
T\left(y^{\prime}\right)-T(y)=T\left(h\left(x^{\prime}\right)\right)-T(h(x))=h\left(f\left(x^{\prime}\right)\right)-h(f(x))=h\left(x^{\prime}\right)-h(x)=y^{\prime}-y,
$$

proving that $\left.T\right|_{\left(y_{\ell-1}, y_{\ell}\right)}$ is a translation. In particular, $\left.T\right|_{\left(y_{\ell-1}, y_{\ell}\right)}$ is injective and $T\left(\left(y_{\ell-1}, y_{\ell}\right)\right)$ is an open interval for each $1 \leq \ell \leq m$. Moreover, since $h$ is order-preserving, if $\ell \neq k$, then 
$h\left(x_{i_{\ell}-1}, x_{i_{\ell}}\right)$ and $h\left(x_{i_{k}-1}, x_{i_{k}}\right)$ are non-overlapping open intervals, implying that $T$ is (globally) injective on $I \backslash\left\{y_{0}, y_{1}, \ldots, y_{m}\right\}$. As for the definition of $T$ on the set $\left\{y_{1}, \ldots, y_{n-1}\right\}$, we can choose one of the lateral limits of $f$ as we approach each of these points in such a way that $T$ is, indeed, globally injective. In this way, $T$ is a $m$-IET.

We claim that $T$ has no attractive periodic orbit. In fact, if for each $1 \leq j \leq s, \gamma_{i}$ is an infinite $f$-orbit dense in $\Lambda_{i}$, then the union of the infinite $T$-orbits $T\left(\gamma_{1}\right), \ldots, T\left(\gamma_{s}\right)$ is a dense subset of $I$, ruling out attractive periodic $T$-orbits.

Let $x \in I$ be a point whose natural $f$-coding is $\theta=\theta_{0} \theta_{1} \ldots$, then we may assume that $\omega(x)$ is infinite, otherwise Lemma 3.1 says that $\theta$ would be ultimately periodic (i.e. $\exists k \geq 0$ such that the natural $f$-coding of $f^{k}(x)$ is periodic). By Corollary 3.4 , there exists $k_{0} \geq 0$ such that $f^{k}(x) \in\left(x_{i_{1}-1}, x_{i_{1}}\right) \cup \cdots \cup\left(x_{i_{m}-1}, x_{i_{m}}\right)$ for all $k \geq k_{0}$. This means that the natural $f$-coding $\zeta=\zeta_{0} \zeta_{1} \ldots$ of $f^{k_{0}}(x)$ is an infinite word over the alphabet $\mathcal{A}^{\prime}=\left\{i_{1}, \ldots, i_{m}\right\}$. Let $\eta=\eta_{0} \eta_{1} \ldots$ be the natural $T$-coding of $y=h(x)$, then $\zeta_{j}=i_{\ell} \in\left\{i_{1}, \ldots, i_{m}\right\}$ if and only if $\eta_{j}=\ell \in\{1, \ldots, m\}$, proving that $\zeta$ and $\eta$ are isomorphic infinite words.

\section{Proofs of Theorem 2.2 and Corollary 2.5}

Proof of Theorem 2.2. Let $T: I \rightarrow I$ be a topologically transitive $n$-IET and $J_{1}, \ldots, J_{n}$ be the associated partition. Without loss of generality we may assume that the endpoints of $J_{i}$ are $y_{i-1}$ and $y_{i}$, where $0=y_{0}<y_{1}<\cdots<y_{n}=1$. Let $\left\{p_{k}\right\}_{k=1}^{\infty} \subset I \backslash\left\{y_{0}, y_{1}, \ldots, y_{n-1}\right\}$ be a dense $T$-orbit. Given $k \geq 1$, let

$$
\mathcal{L}_{k}=\left\{\ell \geq 1: p_{\ell}<p_{k}\right\} \quad \text { and } \quad G_{k}=\left[\sum_{\ell \in \mathcal{L}_{k}} 2^{-\ell}, 2^{-k}+\sum_{\ell \in \mathcal{L}_{k}} 2^{-\ell}\right] .
$$

Notice that $p_{k}>0$ and $\mathcal{L}_{k} \neq \emptyset$. Hence, $G_{k} \subset(0,1)$ is a well-defined interval of length $\left|G_{k}\right|=2^{-k}$. We claim that $\left\{p_{k}\right\}_{k \geq 1}$ and $\left\{G_{k}\right\}_{k \geq 1}$ share the same ordering meaning that

$$
p_{k}<p_{j} \Longleftrightarrow \sup G_{k}<\inf G_{j} .
$$

In fact, $p_{k}<p_{j}$ if and only if $\{k\} \cup \mathcal{L}_{k} \subset \mathcal{L}_{j}$, which is equivalent to

$$
\sup G_{k}=2^{-k}+\sum_{\ell \in \mathcal{L}_{k}} 2^{-\ell}<\sum_{\ell \in \mathcal{L}_{j}} 2^{-\ell}=\inf G_{j}
$$

In particular, we have that the intervals $G_{1}, G_{2}, \ldots$ are pairwise disjoint and their union is dense because $\sum_{k=1}^{\infty}\left|G_{k}\right|=1$. Applying (8) we conclude that if $J \subset I$ is an interval and

$$
\left\{m_{k}\right\}_{k \geq 1}=\left\{\ell \geq 1: p_{\ell} \in J\right\}, \quad \text { then } \overline{\cup_{k \geq 1} G_{m_{k}}} \text { is an interval. }
$$

Let $\widehat{h}: \cup_{k \geq 1} G_{k} \rightarrow I$ be the function that on $G_{k}$ takes the constant value $p_{k}$. By (8), we have that $\widehat{h}$ is nondecreasing and has dense domain and dense range. Thus, $\widehat{h}$ admits a unique nondecreasing continuous surjective extension $h:[0,1] \rightarrow[0,1]$ to the whole interval $[0,1]$. It is elementary to see that $h^{-1}\left(\left\{p_{k}\right\}\right)=G_{k}$. Denote by $I_{1}, \ldots, I_{n}$ the partition of $I$ defined by $I_{i}=h^{-1}\left(J_{i}\right)$. Notice that $x_{i}=h^{-1}\left(y_{i}\right), 0 \leq i \leq n$, are the endpoints of the partition $I_{1}, \ldots, I_{n}$. 
Let $\widehat{f}: \cup_{k \geq 1} G_{k} \rightarrow \cup_{k \geq 2} G_{k}$ be such that $\left.\widehat{f}\right|_{G_{k}}: G_{k} \rightarrow G_{k+1}$ is an affine bijection with slope $\frac{1}{2} T^{\prime}\left(p_{k}\right)$ for every $k \geq 1$, where $T^{\prime}\left(p_{k}\right) \in\{-1,1\}$ is the derivative of $T$ at $p_{k}$. We claim that for each $1 \leq i \leq n$, there exist a dense subset $\widehat{I}_{i}$ of $I_{i}, \lambda_{i} \in\left\{-\frac{1}{2}, \frac{1}{2}\right\}$ and $b_{i} \in \mathbb{R}$ such that

$$
\widehat{f}(x)=\lambda_{i} x+b_{i} \quad \text { for all } \quad x \in \widehat{I}_{i}
$$

In order to show that (9) is true, fix $1 \leq i \leq n$ and let $\left\{m_{k}\right\}_{k \geq 1}=\left\{\ell \geq 1: p_{\ell} \in J_{i}\right\}$, then $\widehat{J}_{i}=\cup_{k \geq 1}\left\{p_{m_{k}}\right\}$ is a dense subset of $J_{i}$ and $\widehat{I}_{i}=\cup_{k \geq 1} G_{m_{k}}$ is a dense subset of $I_{i}$. Moreover, there exists $\lambda_{i} \in\left\{-\frac{1}{2}, \frac{1}{2}\right\}$ such that $T^{\prime}(y)=2 \lambda_{i}$ for all $y \in J_{i}$. In particular, $T^{\prime}\left(p_{m_{k}}\right)=2 \lambda_{i}$ for all $k \geq 1$. By definition, $\left.\widehat{f}\right|_{G_{m_{k}}}: G_{m_{k}} \rightarrow G_{m_{k}+1}$ is an affine bijection with slope $\frac{1}{2} T^{\prime}\left(p_{m_{k}}\right)=\lambda_{i}$ for all $k \geq 1$, which proves (9). We have proved that there exist $\lambda_{i} \in\left\{-\frac{1}{2}, \frac{1}{2}\right\}$ and $c_{m_{k}} \in \mathbb{R}$ such that

$$
\widehat{f}(x)=\lambda_{i} x+c_{m_{k}} \quad \text { for all } \quad x \in G_{m_{k}} .
$$

Let us prove that if $\lambda_{i}=\frac{1}{2}$ (respectively, $\lambda_{i}=-\frac{1}{2}$ ) then $\widehat{f}$ is strictly increasing (respectively, strictly decreasing) on $\cup_{k \geq 1} G_{m_{k}}$. Without loss of generality, assume that $\lambda_{i}=-\frac{1}{2}$, then $\widehat{f}$ is strictly decreasing on each interval $G_{m_{k}}$. Let $y_{k}<z_{j}$ be such that $y_{k} \in G_{m_{k}}$ and $z_{j} \in G_{m_{j}}$, where $k \neq j$ and $\sup G_{m_{k}}<\inf G_{m_{j}}$. By (8), we have that $p_{m_{k}}<p_{m_{j}}$ and $\left\{p_{m_{k}}, p_{m_{j}}\right\} \subset J_{i}$. Then, since $T^{\prime}(y)=2 \lambda_{i}=-1$ for all $y \in J_{i}$, we have that $\left.T\right|_{J_{i}}$ is decreasing, thus $T\left(p_{m_{k}}\right)>T\left(p_{m_{j}}\right)$, that is, $p_{m_{k}+1}>p_{m_{j}+1}$. By (8) once more, we get $\sup G_{m_{j}+1}<\inf G_{m_{k}+1}$. By definition, $f\left(y_{k}\right) \in G_{m_{k}+1}$ and $f\left(z_{j}\right) \in G_{m_{j}+1}$, thus $f\left(y_{k}\right)>f\left(z_{j}\right)$. This proves that $\widehat{f}$ is decreasing on $\cup_{k \geq 1} G_{m_{k}}$. It remains to prove that $c_{m_{k}}$ in (10) is the same for all $k \geq 1$. Let $j \neq k$. We may assume that $a=\sup G_{m_{j}}<\inf G_{m_{k}}=b$. Notice that

$$
\begin{aligned}
\frac{1}{2}(b-a)+\frac{\left|\lambda_{i}\right|}{\lambda_{i}}\left(c_{m_{k}}-c_{m_{j}}\right) & =\frac{\left|\lambda_{i}\right|}{\lambda_{i}}(\widehat{f}(b)-\widehat{f}(a))=\sum_{G_{m_{\ell}} \subset[a, b]}\left|\widehat{f}\left(G_{m_{\ell}}\right)\right| \\
& =\frac{1}{2} \sum_{G_{m_{\ell}} \subset[a, b]}\left|G_{m_{\ell}}\right|=\frac{1}{2}(b-a)
\end{aligned}
$$

yielding $c_{m_{k}}=c_{m_{j}}$. Thus, (9) is true.

It follows from (9) that $\left.\widehat{f}\right|_{\cup_{k \geq 1} G_{m_{k}}}$ admits a unique monotone continuous extension to the interval $I_{i}=h^{-1}\left(J_{i}\right)$. This extension is also an affine map with slope equal to $\frac{1}{2}$ in absolute value. Since $i$ is arbitrary, we obtain an injective piecewise $\frac{1}{2}$-affine extension $f$ of $\widehat{f}$ to the whole interval $I=\cup_{i=1}^{n} I_{i}$.

It remains to show that $h \circ f=T \circ h$. In fact, for every $y \in G_{k}$, we have that

$$
h(f(y))=\widehat{h}(\widehat{f}(y))=p_{k+1}=T\left(p_{k}\right)=T(\widehat{h}(y))=T(h(y)) .
$$

Hence, (11) holds for a dense set of $y \in I$. By continuity, (11) holds for every $y \in I$. 
Proof of Corollary 2.5. Let $0<\alpha<1$ be irrational. Let $T: I \rightarrow I$ be the 2-IET defined by $T(y)=y+\alpha(\bmod 1)$, or equivalently, let $J_{1}=[0,1-\alpha), J_{2}=[1-\alpha, 1)$, and

$$
T(y)=\left\{\begin{array}{lll}
y+\alpha & \text { if } & y \in J_{1} \\
y+\alpha-1 & \text { if } & y \in J_{2}
\end{array} .\right.
$$

It is widely known that $T$ is minimal. Hereafter, we take all the notation of the proof of Theorem 2.2. Let $y_{0}=0, y_{1}=1-\alpha$ and $y_{2}=1$. Let $\gamma=\left\{p_{k}\right\}_{k=1}^{\infty}=\{\alpha, T(\alpha), \ldots\}$ be the $T$-orbit of $\alpha$, then $\gamma$ is a dense orbit contained in $I \backslash\left\{y_{0}, y_{1}\right\}$. Let $\theta=\theta_{0} \theta_{1} \ldots$ be the natural $T$-coding of $\alpha$, then $\theta$ is a Sturmian word. Let us define the 2-PC $f_{T}$. Let $G_{k}, k \geq 1$, be the pairwise disjoint intervals of length $\left|G_{k}\right|=2^{-k}$ defined by (7). Let $I_{i}=h^{-1}\left(J_{i}\right)$ for $i=1,2$, then $I_{1}=\left[0, x_{1}\right), I_{2}=\left[x_{1}, 1\right)$, where $x_{1}=h^{-1}\left(y_{1}\right)$. Let

$$
\left\{m_{k}\right\}_{k \geq 1}=\left\{\ell \geq 1: p_{\ell} \in J_{1}\right\}=\left\{\ell \geq 1: \theta_{\ell-1}=1\right\}
$$

then $\widehat{J_{1}}=\cup_{k \geq 1}\left\{p_{m_{k}}\right\}$ is a dense subset of $J_{1}$ and $\widehat{I_{1}}=\cup_{k \geq 1} G_{m_{k}}$ is a dense subset of $I_{1}$. In this way, since $\left|G_{m_{k}}\right|=2^{-m_{k}}$, we have that

$x_{1}=\sup I_{1}=\sum_{k \geq 1}\left|G_{m_{k}}\right|=\sum_{k \geq 1} 2^{-m_{k}}=\sum_{\ell \geq 1}\left(2-\theta_{\ell-1}\right) 2^{-\ell}=\frac{1}{2} \sum_{\ell \geq 0}\left(2-\theta_{\ell}\right) 2^{-\ell}=2-\frac{1}{2} \sum_{\ell \geq 0} \theta_{\ell} 2^{-\ell}$.

Since $T^{\prime}(y)=1$ for every $y \in I$, we have that the slope $\lambda_{i}$ of $f_{T}$ is $\frac{1}{2}$. In this way, we have that

$$
f_{T}(x)=\left\{\begin{array}{lll}
\frac{1}{2} x+b_{1} & \text { if } & x \in\left[0, x_{1}\right) \\
\frac{1}{2} x+b_{2} & \text { if } & x \in\left[x_{1}, 1\right)
\end{array} .\right.
$$

Since $\frac{1}{2} x_{1}+b_{1}=1$ and $\frac{1}{2} x_{1}+b_{2}=0$, we conclude that

$$
f_{T}(x)=\frac{1}{2} x+\delta, \quad \text { where } \quad \delta=\frac{1}{4} \sum_{\ell \geq 0} \theta_{\ell} 2^{-\ell} .
$$

It is clear that

$$
\delta=\frac{1}{4}\left(1+\theta_{0}+\sum_{\ell \geq 1}\left(\theta_{\ell}-1\right) 2^{-\ell}\right)
$$

thus $\left\{\theta_{\ell}-1\right\}_{\ell \geq 1}$ is the binary expansion of $\sum_{\ell \geq 1}\left(\theta_{\ell}-1\right) 2^{-\ell}$. In this case, the transcendence of $\delta$ follows from Ferenczi and Mauduit [13, Proposition 2] or Adamczewski and Cassaigne [1, Theorem 1] together with the fact that $w=\left(\theta_{1}-1\right)\left(\theta_{2}-2\right) \ldots$ is a Sturmian word.

Now let us consider the particular case in which $\alpha=2-\varphi$, where $\varphi=(1+\sqrt{5}) / 2$ is the golden ratio. In this case, it is known that $\left(\theta_{1}-1\right)\left(\theta_{2}-1\right) \ldots$ is the Fibonacci word

$$
\theta-1=010010100100101001010010010100100101001010010010100 \ldots
$$

The number

$$
R=1-\sum_{\ell \geq 0}\left(\theta_{\ell}-1\right) 2^{-(\ell+1)}=0.7098034428612913146 \ldots
$$


is known in the mathematical literature as the rabbit constant. Notice that by (12), we have that

$$
\delta=\frac{1}{4}\left(1+\theta_{0}+2 \sum_{\ell \geq 1}\left(\theta_{\ell}-1\right) 2^{-(\ell+1)}\right)=\frac{1}{4}\left(2+2 \sum_{\ell \geq 0}\left(\theta_{\ell}-1\right) 2^{-(\ell+1)}\right)=1-\frac{R}{2} .
$$

The transcendence of the rabbit constant was proved by Davison [9].

\section{Proofs of Corollary 2.3 and Corollary 2.4}

Proof of Corollary 2.3. Let $\theta=\theta_{0} \theta_{1} \ldots$ be a natural coding of an injective $n$-PC $f: I \rightarrow I$. By Theorem 2.1, there exist a $m$-IET $T: I \rightarrow I$ with $2 \leq m \leq n$ and $q \geq 0$ such that the infinite word $\theta^{*}=\theta_{q} \theta_{q+1} \ldots$ is either periodic or isomorphic to the non ultimately periodic natural $T$-coding $\omega=\omega_{0} \omega_{1} \ldots$ of some point $y \in I$. For the sake of simplification, we will only consider the case in which $T$ is an orientation-preserving $m$-IET with associated partition $I_{1}=\left[y_{0}, y_{1}\right), \ldots, I_{m}=\left[y_{m-1}, y_{m}\right)$. Since $\omega$ is non ultimately periodic, there exist $r \geq 0$ and $y^{*}=T^{r}(y)$ whose $T$-orbit is regular, which means

$$
O_{T}\left(y^{*}\right)=\left\{T^{r}(y), T^{r+1}(y), \ldots\right\} \subset I \backslash\left\{y_{0}, y_{1}, \ldots, y_{m-1}\right\} .
$$

Because $O_{T}(y *)$ is regular, it is entirely contained in a minimal component of $T$. More specifically, there exist open intervals $A_{1}, \ldots, A_{p}$ with pairwise disjoint closures such that $O_{T}\left(y^{*}\right)$ is a dense subset of $A_{1} \cup \cdots \cup A_{p}$ and $T\left(A_{1}\right) \subset A_{2}, \ldots, T\left(A_{p-1}\right) \subset A_{p}, T\left(A_{p}\right) \subset A_{1}$, and $T$ takes $I \backslash\left(\overline{A_{1}} \cup \cdots \cup \overline{A_{p}}\right)$ into itself (see [3, 24]). Let $\mu$ be the normalized Lebesgue measure on $A_{1} \cup \cdots A_{p}$, then $\mu$ is $T$-invariant: $\mu\left(T^{-1}(B)\right)=\mu(B)$ for every Borel set $B \subset I$. Let $h:[0,1] \rightarrow[0,1]$ be the nondecreasing, continuous, surjective map defined by $h(t)=\mu([0, t]), t \in I$. Notice that $h$ is strictly increasing on $A_{1} \cup \cdots \cup A_{p}$ and constant on each of the finitely many connected components of $I \backslash A_{1} \cup \cdots \cup A_{p}$. Given $y, y^{\prime} \in I_{i}$ with $h(y)=h\left(y^{\prime}\right)$, we claim that $h(T(y))=h\left(T\left(y^{\prime}\right)\right)$. Without loss of generality, assume that $y \leq y^{\prime}$, then $T(y) \leq T\left(y^{\prime}\right)$. Moreover, since $\left.T\right|_{I_{i}}$ is a translation,

$$
\left[y, y^{\prime}\right]=T^{-1}\left(\left[T(y), T\left(y^{\prime}\right)\right]\right) .
$$

Hence, since $\mu$ is non-atomic and $T$-invariant, we have that for any $y, y^{\prime} \in I_{i}$,

$$
h\left(T\left(y^{\prime}\right)\right)-h(T(y))=\mu\left(\left[T(y), T\left(y^{\prime}\right)\right]\right)=\mu\left(T^{-1}\left(\left[T(y), T\left(y^{\prime}\right)\right]\right)\right)=h\left(y^{\prime}\right)-h(y),
$$

which proves the claim.

We will use (13) to define an IET $E: I \rightarrow I$. Let

$$
\mathcal{I}=\left\{1 \leq i \leq m: I_{i} \cap\left(A_{1} \cup \cdots \cup A_{p}\right) \neq \emptyset\right\}
$$

Let $m^{\prime} \leq m$ be the cardinality of $\mathcal{I}$, then we may write $\mathcal{I}=\left\{i_{1}, \ldots, i_{m^{\prime}}\right\}$. The intervals $J_{1}=h\left(I_{i_{1}}\right), \ldots, J_{m^{\prime}}=h\left(I_{i_{m^{\prime}}}\right)$ form a partition of $I$ into non-degenerate intervals with 
endpoints $0=z_{0}<z_{1}<\cdots<z_{m^{\prime}}=1$ defined by $z_{\ell}=h\left(y_{i_{\ell}}\right), 0 \leq \ell \leq m^{\prime}$. Let $E: I \rightarrow I$ be the right-continuous map that at $z=h(y) \in I \backslash\left\{z_{0}, z_{1}, \ldots, z_{m^{\prime}-1}\right\}$ takes the value

$$
E(h(y))=h(T(y))
$$

The map $E$ is well-defined. In fact, if $y, y^{\prime} \in I$ are such that $h(y)=h\left(y^{\prime}\right)$, then $y, y^{\prime}$ belong to the same connected component of $I \backslash\left(A_{1} \cup \cdots \cup A_{p}\right)$. There is no discontinuity of $T$ between $y$ and $y^{\prime}$, otherwise $h(y)$ would belong to $\in\left\{z_{1}, \ldots, z_{m^{\prime}-1}\right\}$. In this way, $y, y^{\prime}$ belong to the same interval $I_{i}$ and (13) asserts that $E$ is well-defined. Notice that, by definition, $E\left(z_{\ell}\right)=\lim _{\epsilon \rightarrow 0^{+}} E\left(z_{\ell}+\epsilon\right)$ for all $0 \leq \ell \leq m^{\prime}-1$.

Let us prove that $\left.E\right|_{\left(z_{\ell-1}, z_{\ell}\right)}, 1 \leq \ell \leq m^{\prime}$, is a translation. If $z, z^{\prime}$ are two points in $\left(z_{\ell-1}, z_{\ell}\right)$, then there exist $y, y^{\prime} \in\left(y_{i_{\ell-1}}, y_{i_{\ell}}\right)$ such that $z=h(y)$ and $z^{\prime}=h\left(y^{\prime}\right)$. Now (13) and (14) yield

$$
E\left(z^{\prime}\right)-E(z)=E\left(h\left(y^{\prime}\right)\right)-E(h(y))=h\left(T\left(y^{\prime}\right)\right)-h(T(y))=h\left(y^{\prime}\right)-h(y)=z^{\prime}-z,
$$

proving that $\left.E\right|_{J_{\ell}}$ is a translation.

The map $E$ is surjective. In fact, since $h$ and $T$ are surjective, given $z \in I$, there exists $y \in I$ such that $E(h(y))=h(T(y))=z$. To see that $E$ is also injective, by the above, $E$ takes each interval $J_{\ell}$ into its translate $E\left(J_{\ell}\right)$, which therefore has the same length, that is, $\left|E\left(J_{\ell}\right)\right|=\left|J_{\ell}\right|$. Since $E$ is surjective, we have that

$$
1=\sum_{\ell=1}^{m^{\prime}}\left|E\left(J_{\ell}\right)\right| \leq \sum_{\ell=1}^{m^{\prime}}\left|J_{\ell}\right| \leq 1,
$$

implying that no overlapping is possible for the intervals $E\left(J_{1}\right), \ldots, E\left(J_{m^{\prime}}\right)$. This proves that $E$ is a $m^{\prime}$-IET.

Becasuse $O_{T}\left(y^{*}\right)$ is a dense subset of $A_{1} \cup \cdots \cup A_{p}$ and $h\left(A_{1} \cup \cdots \cup A_{p}\right)$ is dense in $I$, we have that $h$ takes the $T$-orbit $O_{T}\left(y^{*}\right)$ onto a dense $E$-orbit, thus $E$ is topologically transitive. Moreover, if $\zeta=\zeta_{0} \zeta_{1} \ldots$ is the natural $T$-coding of $y^{*}$ and $\eta=\eta_{0} \eta_{1} \ldots$ is the natural $E$-coding of $z^{*}=h\left(y^{*}\right)$, then $\zeta_{k}=i_{\ell} \in\left\{i_{1}, \ldots, i_{m^{\prime}}\right\}$ if and only if $\eta_{k}=\ell \in$ $\left\{1, \ldots, m^{\prime}\right\}$, proving that $\zeta$ and $\eta$ are isomorphic infinite words. To conclude the proof, we recall that $\theta_{q+r} \theta_{q+r+1} \ldots$ is isomorphic to $\zeta$.

Lemma 6.1. Let $\theta=\theta_{0} \theta_{1} \ldots$ be an infinite word and $\theta^{*}=\theta_{q+1} \theta_{q+2} \ldots$ an infinite subword of $\theta$, then there exist $k_{0} \geq 1$ and $\beta \geq 0$ such that

$$
p_{k}(\theta)=p_{k}\left(\theta^{*}\right)+\beta \quad \text { for every } \quad k \geq k_{0} .
$$

Proof. For each $k \geq q+1$, let

$$
\begin{aligned}
\mathcal{W}_{k} & =\left\{\theta_{0} \theta_{1} \ldots \theta_{k-1}, \quad \theta_{1} \theta_{2} \ldots \theta_{k}, \quad \ldots, \quad \theta_{q} \theta_{q+1} \ldots \theta_{q+k-1}\right\} \subset L_{k}(\theta) \\
\mathcal{W}_{k}^{*} & =\left\{\omega \in \mathcal{W}_{k}: \omega \notin L_{k}\left(\theta^{*}\right)\right\}
\end{aligned}
$$


Notice that $\mathcal{W}_{k}$ is formed by at most $q+1$ distinct finite words and $k \mapsto \# \mathcal{W}_{k}^{*}$ is a nondecreasing map, thus there exist $k_{0} \geq 0$ and $\beta \leq q+1$ such that $\# \mathcal{W}_{k}=\beta$ for every $k \geq k_{0}$. Moreover, for every $k \geq k_{0}$, we have the disjoint union

$$
L_{k}(\theta)=\mathcal{W}_{k}^{*} \cup L_{k}\left(\theta^{*}\right), \quad \text { thus } \quad p_{k}(\theta)=p_{k}\left(\theta^{*}\right)+\beta \text {. }
$$

Lemma 6.2. Let $\theta$ be a natural coding of a topologically transitive $m$-IET T:I $\rightarrow I$, then there exist $k_{0} \geq 1, \alpha \in\{0, \ldots, m-1\}$ and $\beta \geq 1$ such that

$$
p_{\theta}(k)=k \alpha+\beta \quad \text { for every } \quad k \geq k_{0} .
$$

Moreover, if $T$ is a standard $m$-IET, with $m \geq 2$, satisfying the i.d.o.c., then $\alpha=m-1$, $\beta=1$ and $k_{0}=1$.

Proof. Let $T: I \rightarrow I$ be a topologically transitive $m$-IET and $\mathscr{P}=\left\{I_{1}, \ldots, I_{m}\right\}$ be the partition associated to $T$, then, since $T^{-1}$ is also an IET, $T^{-k}(\mathscr{P})$ is a partition of $I$ into intervals for every $k \geq 0$, implying that the members of the set

$$
\mathscr{P}_{k}=\bigwedge_{\ell=0}^{k-1} T^{-\ell}(\mathscr{P})=\left\{I_{i_{0}} \cap T^{-1}\left(I_{i_{1}}\right) \cap \cdots \cap T^{-(k-1)}\left(I_{i_{k-1}}\right): 1 \leq i_{0}, i_{1}, \ldots, i_{k-1} \leq m\right\} .
$$

are pairwise disjoint intervals. Moreover, if $\theta$ is a natural coding of $T$, then the $k$-word $i_{0} i_{1} \ldots i_{k-1}$ occurs in $\theta$ if and only if the interval $J=I_{i_{0}} \cap T^{-1}\left(I_{i_{1}}\right) \cap \cdots \cap T^{-(k-1)}\left(I_{i_{k-1}}\right) \in$ $\mathscr{P}_{k}$ is nom-empty.

Let $\theta$ be the natural $T$-coding of some point $x \in I$. If $\theta$ is (ultimately) periodic, then by the Morse-Hedlund Theorem, there exist $k_{0} \geq 1$ and $\beta \geq 1$ such that $p_{\theta}(k)=\beta$ for every $k \geq k_{0}$, meaning that (15) holds with $\alpha=0$. Hence, we may assume that $\theta$ is not (ultimately) periodic. In this case, there exists $q \geq 0$ such that the orbit $\left\{x^{*}, T\left(x^{*}\right), \ldots\right\}$ of $x^{*}=T^{q+1}(x)$ is a dense subset of $I \backslash\left\{x_{0}, x_{1}, \ldots, x_{m-1}\right\}$, where $0=x_{0}<x_{1}<\cdots<x_{m}=1$ are the endpoints of the partition $\mathscr{P}$. In this way, for each $k \geq 1,\left\{x^{*}, T\left(x^{*}\right), \ldots\right\}$ is contained in the union of the interiors of the intervals of $\mathscr{P}_{k}$. Hence, the $k$-word $i_{0} i_{1} \ldots i_{k-1}$ occurs in the natural $T$-coding $\theta^{*}$ of $x^{*}$ if and only if the interval $J=I_{i_{0}} \cap T^{-1}\left(I_{i_{1}}\right) \cap \cdots \cap$ $T^{-(k-1)}\left(I_{i_{k-1}}\right) \in \mathscr{P}_{k}$ has non-empty interior. Therefore, the number of such intervals $J$ in $\mathscr{P}_{k}$ equals $p_{k}\left(\theta^{*}\right)$ and is related to the number of endpoints of the partition $\mathscr{P}_{k}$ as follows

$$
p_{k}\left(\theta^{*}\right)=1+\sum_{\ell=0}^{k-1} m_{\ell}
$$

where $m_{0}=m-1$ and

$$
m_{\ell}=\left\{T^{-\ell}\left(x_{1}\right), \ldots, T^{-\ell}\left(x_{m-1}\right)\right\} \backslash \bigcup_{p=0}^{\ell-1}\left\{T^{-p}\left(x_{1}\right), \ldots, T^{-p}\left(x_{m-1}\right)\right\}
$$

gives the number of new division points at the $\ell$-th step towards the construction of $\mathscr{P}_{k}$. The map $\ell \mapsto m_{\ell}$ is a non-increasing, therefore there exist $k_{0}^{\prime} \geq 0$ and $\alpha \geq 1$ such that 
$m_{\ell}=\alpha$ for every $\ell \geq k_{0}^{\prime}$. Notice that $\alpha \geq 1$ because, as $\theta^{*}$ is not (ultimately) periodic, $p_{k}\left(\theta^{*}\right) \rightarrow \infty$ as $k \rightarrow \infty$. Let $\beta_{0}, \beta_{1}, \ldots, \beta_{k_{0}-1} \geq 0$ be such that

$$
m_{\ell}=\left\{\begin{array}{ll}
\alpha+\beta_{\ell} & \text { if } \quad \ell \in\left\{0,1, \ldots, k_{0}^{\prime}-1\right\} \\
\alpha & \text { if } \quad \ell \geq k_{0}^{\prime}
\end{array} .\right.
$$

By (16) and (17), we have that if $\beta^{\prime}=1+\beta_{0}+\beta_{1}+\cdots+\beta_{k_{0}-1}$, then

$$
p_{k}\left(\theta^{*}\right)=1+\sum_{\ell=0}^{k_{0}^{\prime}-1}\left(\alpha+\beta_{\ell}\right)+\sum_{k_{0}^{\prime}}^{k-1} \alpha=\alpha k+\beta^{\prime} \quad \text { for all } \quad k \geq k_{0}^{\prime}+1 .
$$

By Lemma 6.1, there exist $k_{0} \geq k_{0}^{\prime}+1$ and $\beta^{\prime \prime} \geq 0$ such that

$$
p_{k}(\theta)=p_{k}\left(\theta^{*}\right)+\beta^{\prime \prime}=\alpha k+(\underbrace{\beta^{\prime}+\beta^{\prime \prime}}_{\beta})=\alpha k+\beta \quad \text { for all } \quad k \geq k_{0} .
$$

Notice that if $T$ satisfies the i.d.o.c., then $\theta^{*}=\theta$ and $m_{\ell}=m-1$ for all $\ell \geq 0$, then (16) yields

$$
p_{k}(\theta)=p_{k}\left(\theta^{*}\right)=(m-1) k+1 \quad \text { for all } \quad k \geq 1,
$$

implying that in this case (15) holds with $\alpha=m-1, \beta=1$ and $k_{0}=1$.

Proof of Corollary 2.4. Let $f: I \rightarrow I$ be an injective $n$-PC and $\theta=\theta_{0} \theta_{1} \ldots$ be the natural $f$-coding of $x \in I$. By Corollary [2.3, there exist $k \geq 0$ and a topologically transitive $m$-IET, with $2 \leq m \leq n$, such that the natural coding $\theta^{*}$ of $f^{k}(x)$ is either periodic or isomorphic to a non ultimately periodic natural coding of $T$. By Lemma 6.2, there exist $k_{0} \geq 1, \alpha \in\{0, \ldots, m-1\}$ and $\beta \geq 1$ such that

$$
p_{k}\left(\theta^{*}\right)=k \alpha+\beta \quad \text { for all } \quad k \geq k_{0} .
$$

Notice that in the case in which $\theta^{*}$ is periodic, by the Morse-Hedlund Theorem, (18) holds with $\alpha=0$. To conclude the proof of the item $(i)$, apply Lemma 6.1. As for tye item $(i i)$, we apply Theorem 2.2 together with Lemma 6.2.

\section{REFERENCES}

[1] B. Adamczewski and J. Cassaigne, On the transcendence of real numbers with a regular expansion, J. Number Theory 103 (2003) 27-37.

[2] A. Ya. Belov and A. L. Chernyat'ev, Words with low complexity and interval exchange transformations Communications of the Moscow Mathematical Society, Russian Mathematical Surveys 63(1) (2008) 158-160.

[3] M. Boshernitzan, Rank two interval exchange transformations, Ergodic Theory Dynam. Systems 8(1988) 379-394.

[4] Y. Bugeaud, Dynamique de certaines applications contractantes linéaires par morceaux, sur [0, 1), C. R. Math. Acad. Sci. Paris Ser. I Math. 317 (6) (1993) 575-578.

[5] Y. Bugeaud, Linear mod one transformations and the distribution of fractional parts of $\xi(p / q)^{n}$, Acta Arith. 114 (4) (2004) 301-311.

[6] Y. Bugeaud and J.-P. Conze. Calcul de la dynamique de transformations linéaires contractantes mod 1 et arbre de Farey, Acta Arith. 88 (3) (1999) 201-218. 
[7] E. Catsigeras, P. Guiraud and A. Meyroneinc, Complexity of injective piecewise contracting interval maps, Ergodic Theory Dynam. Systems (2018) 1-25, doi:10.1017/etds.2018.34

[8] T. Cherry, Analytic quasi-periodic curves of discontinuous type on a torus, Proc. Lond. Math. Soc. 44 (1938) 175-215.

[9] J. L. Davison, A series and its associated continued fraction, Proc. Amer. Math. Soc. 63(1)(1977) 29-32.

[10] F. Dolce and D. Perrin, Interval exchanges, admissibility and branching Rauzy induction, RAIROTheor. Inf. Appl. 51 (2017) 141-166.

[11] S. Ferenczi, Combinatorial methods for interval exchange transformations, Southeast Asian Bull. Math. 37 (1) (2013) 47-66.

[12] S. Ferenczi and L. Q. Zamboni, Languages of k-interval exchange transformations, Bull. London Math. Soc. 40 (2008) 705-714.

[13] S. Ferenczi and C. Mauduit, Transcendence of numbers with a low complexity expansion, J. Number Theory 67 (1997) 146-161.

[14] J-M. Gambaudo and C. Tresser, On the dynamics of quasi-contractions, Bull. Braz. Math. Soc. $19(1)(1988) 61-141$.

[15] J-M. Gambaudo and C. Tresser, Dynamique régulière ou chaotique: applications du cercle ou de l'intervalle ayant une discontinuité, C.R. Acad. Sci. Paris Ser. I Math 300 (1985) 311-313.

[16] S. Janson and A. Öberg, A piecewise contractive dynamical system and election methods, https://arxiv.org/pdf/1709.06398.pdf

[17] M. Keane, Interval exchange transformations, Math Z 141(1)(1975) 25-31.

[18] M. Laurent and A. Nogueira, Rotation number of interval contracted rotations, Journal of Modern Dynamics 12(2018).

[19] L-M. Lopez and P. Narbel, Substitutions and interval exchange transformations of rotation class, Theoret. Comput. Sci. 255 (2001) 323-344.

[20] A. Nogueira, Almost all interval exchange transformations with flips are nonergodic, Ergodic Theory Dynam. Systems $\mathbf{9}(3)(1989)$ 515-525.

[21] A. Nogueira and B. Pires, Dynamics of piecewise contractions of the interval, Ergodic Theory Dynam. Systems 35 (2015) 2198-2215.

[22] A. Nogueira, B. Pires and R. Rosales, Topological dynamics of piecewise $\lambda$-affine maps, Ergodic Theory Dynam. Systems, 38(5) (2018) 1876-1893.

[23] A. Nogueira, B. Pires and R. Rosales, Asymptotically periodic piecewise contractions of the interval, Nonlinearity 27(7)(2014) 1603-1610.

[24] A. Nogueira, B. Pires and S. Troubetzkoy, Orbit structure of interval exchange transformations with flip, Nonlinearity 26 (2013) 525-537.

[25] K. Parthasarathy, Probability measures on metric spaces, American Mathematical Society, 2005.

[26] B. Pires, Invariant measures for piecewise continuous maps, C. R. Acad. Sci. Paris Ser. I Math, 354 (2016) 717-722. 